concerns, observation is often favored. However, with a growing elderly population, many of whom are in good health, endovascular management of elective aneurysms could be considered. The aim of this study is to analyze clinical outcomes in patients older than 70 years with unruptured cerebral aneurysms treated electively.

Methodology All patients over 70 years of age who were treated electively for unruptured cerebral aneurysms between Jan 2017-Feb 2019 at Westchester Medical Center were included in the study. A retrospective chart review was performed. Demographics, presentation, aneurysm location, clinical complications, and modified Rankin score (mRS) at 30 days were noted. Good outcomes were defined as $\mathrm{mRS}<2$ at 30 -days or discharge to home and bad outcomes were defined as $\mathrm{mRS}>2$ or rehab placement. To compare pre-existing comorbidities, the Charlston Comorbidity Index (CCI) was used.

Results A total of 22 patients were included with a mean age of 76.8 years (range $71-86$ years). The male:female ratio was $1: 6.78 \%$ of the procedures involved flow diversion using the Pipeline Flex device; the remaining 22\% involved coil embolization. There were no neurological complications in our series. Groin complications were seen in 2 patients. The mean CCI for the entire group was 6 and for those with complications was 7. Good outcomes were seen in 95.4\% (21/22) of the patients included in this study.

Conclusion Contrary to popular assumption, good outcomes were seen in more than $95 \%$ of patients above 70 years of age who undergo elective treatment of unruptured cerebral aneurysms. The rate of neurological complications was $0 \%$ in our series. The 2 groin complications noted in this series may be associated with atherosclerotic vasculature and use of dual antiplatelet agents for flow diversion. Given the overall safety and the probability of good outcomes, these findings suggest that patients above 70 years with high-risk unruptured aneurysms should not be denied elective aneurysmal treatment.

Disclosures G. Kaur: None. J. Cooper: None. L. Melathe: None. F. Al-Mufti: None. C. Gandhi: None. J. Santarelli: None.

\section{E-036 UNEXPECTED PROXIMAL STENT MIGRATION DURING THROUGH STENT TECHNIQUE FOR STENT ASSISTED COIL EMBOLIZATION (NEUROFORM ATLAS STENT)}

Y Jung*, H Lee, J Kim, C Chang. Neurovascular surgery/Neurointervention, Daegu, Korea, republic of

\subsection{6/neurintsurg-2019-SNIS.111}

Background and importance When attempting through the stent technique in the stent assisted coiling, there is as risk of the distal stent migration due to the microwire or microcathter. However, we had never seen a case in which a stent slipped downward in the literature review. We report a case of proximal stent migration.

Clinical presentation A 79 - year - old female patient was admitted with a wide neck aneurysm of the middle cerebral artery. The stent-assisted coil was designed to be saving the incorporated branch. With jailing technique, one microcathter was placed in the sac, then the Neuroform Atlas stent was deployed, and another microcatheter was delivered with the through the stent technique for double microcatheter technique. During the re-cross the stent the distal migration was observed. We try removal of the microwire and microcatheter. At the moment of puling the microcatheter and microwire to remove, the existing stent was pulled downward. The patient had a cerebral infarction result in left side motor weakness, can walking with cane.

Discussion The patient was neurologically compromised by unexpected proximal stent migration. Distal stent migration may occur occasionally, but proximal migration is the first experience reported.

Conclusion Physician may remember that unexpected events may occur, and we think that more attention well be needed when doing something. Other reader may wish to remember that this can happen.

Disclosures Y. Jung: None. H. Lee: None. J. Kim: None. C. Chang: None.

\section{E-037 DURABILITY OF TREATMENT FOR THE PENUMBRA SMART COIL SYSTEM AT ONE YEAR IN PATIENTS WITH RUPTURED ANEURYSMS: SUBSET ANALYSIS OF THE SMART REGISTRY}

${ }^{1} \mathrm{D}$ Fiorella, ${ }^{2} \mathrm{~B}$ Bohnstedt, ${ }^{3} \mathrm{C}$ Schirmer, ${ }^{4} \mathrm{R}$ Bellon, ${ }^{5} \mathrm{R}$ Deleacy, ${ }^{6} \mathrm{R}$ Starke, ${ }^{7} \mathrm{~A}$ Spiotta* ${ }^{1}$ Cerebrovascular Center, Stony Brook University Medical Center, Stony Brook, NY; ${ }^{2}$ Oklahoma University Medical Center, OKlahoma City, OK; ${ }^{3}$ Geisinger Medical Center, Danville, PA; ${ }^{4}$ Department of Interventional Neuro Radiology, Swedish Medical Center, Englewood, CO; ${ }^{5}$ The Mount Sinai Hospital, New York, NY; ${ }^{6}$ University of Miami Hospital, Miami, FL; ${ }^{7}$ Medical University of South Carolina, Charleston, SC

\subsection{6/neurintsurg-2019-SNIS.112}

Introduction The Penumbra SMART COIL System includes a novel generation of embolic coils comprised of complex and WAVE shape properties with varying levels of softness to promote dense packing and durable long-term occlusion. We report a subset analysis to assess the durability of treatment with SMART COIL System at one year follow-up in patients with ruptured aneurysms enrolled in the SMART registry.

Materials and methods The SMART registry is a prospective, multi-center registry study. Procedures must employ $\geq 75 \%$ of the SMART, PC400, or POD coils to meet the registry criteria. SMART registry endpoints include retreatment rates through one year follow-up, procedural device-related serious adverse events (SAE), and the ability to achieve adequate occlusion at immediate post-procedure. Multivariate analysis was performed to identify predictors of outcomes at one year. Results Of the first 500 patients with one year follow-up, $24.2 \%(121 / 500)$ of patients had ruptured aneurysms $(74.4 \%$ female; mean age $56.4 \pm 12.7$ years). Ruptured aneurysms were small $(<11 \mathrm{~mm})$ in $89.3 \%(108 / 121)$, large (11 to 25 $\mathrm{mm})$ in $9.9 \%(12 / 121)$, and giant $(>25 \mathrm{~mm})$ in $0.8 \%$ (1/ $121)$ of patients and $30.8 \%(37 / 120)$ were wide-neck (dometo-neck ratio $<1.5$ or neck width $\geq 4 \mathrm{~mm}$ ). Stent-assisted coiling and balloon-assisted coiling were performed in $8.3 \%$ (10/121) and 32.2\% (39/121) of patients, respectively. Median packing density for ruptured aneurysms was $29.7 \%$ (IQR 21.0-40.4).

In patients with ruptured aneurysms, Raymond Class I and II was observed in $90.9 \%(110 / 121)$ at immediate post-procedure and $80.2 \%(89 / 111)$ at one year follow-up. The recanalization rate at one year was $23.4 \%(26 / 111)$. The retreatment 
rate through one year was $14.4 \%$ (17/118). Procedural devicerelated SAE were observed in $4.1 \%$ subjects $(5 / 121)$.

In multivariate models, independent predictors of Raymond Occlusion Class III at one-year follow-up included wide-neck aneurysm (odds ratio $[\mathrm{OR}]=5.69 ; 95 \%$ confidence intervals [CI] 1.69-19.15, $\mathrm{p}=0.0050), \quad$ balloon-assisted coiling $(\mathrm{OR}=3.81 ; 95 \%$ CI $1.28-11.37, \mathrm{p}=0.017)$, and age $\geq 65 \mathrm{yr}$ $(\mathrm{OR}=0.15 ; 95 \%$ CI $0.027-0.78, \mathrm{p}=0.025)$. Balloon-assisted coiling also predicted recanalization at one year follow-up $(\mathrm{OR}=2.54,95 \%$ CI 1.03-6.27, $\mathrm{p}=0.043)$.

Conclusion This subset analysis suggests that the SMART COIL System achieves adequate embolization in ruptured aneurysms with adequate retreatment rates over one year.

Disclosures D. Fiorella: 1; C; Penumbra, Inc. 3; C; Penumbra, Inc. B. Bohnstedt: None. C. Schirmer: None. R. Bellon: 3; C; Penumbra, Inc. R. DeLeacy: None. R. Starke: None. A. Spiotta: 1; C; Penumbra, Inc. 2; C; Penumbra, Inc.

\section{E-038 CORRELATION ANALYSIS OF ENDOVASCULAR TREATMENT FOR RUPTURED INTRACRANIAL ANEURYSM: PREDICTORS FOR UNFAVORABLE OUTCOMES AND RECURRENCE}

${ }^{1} \mathrm{~W}$ Yoon, ${ }^{1} \mathrm{~W}$ Yoon*, ${ }^{1} \mathrm{H}$ Roh, ${ }^{2} \mathrm{~S}$ Suh, ${ }^{1} \mathrm{~T}$ Kwon. ${ }^{1}$ Neurosurgery, Guro hospital, Korea university, Seoul, KOREA, REPUBLIC OF; ${ }^{2}$ Radiology, Guro hospital, Korea university, Seoul, KOREA, REPUBLIC OF

\subsection{6/neurintsurg-2019-SNIS.113}

Purpose The aim of this study is to identify significant predictors of unfavorable outcome and recurrence in the patients treated with endovascular treatment in the setting of ruptured aneurysms.

Method and material A total of 102 ruptured aneurysms of 102 patients who were treated with endovascular treatment including stent-assisted coil embolization and coil embolization without stent were enrolled in this study. In order to identify significant predictors of unfavorable outcome and recurrence, retrospective correlation analysis was performed.

Result A total of 72 patients (70.59\%) showed favorable outcome (mRS 0-2) at their last follow up day and a total of 30 patients (29.41\%) showed unfavorable outcome (mRS 3-6). On univariate and multivariate analysis for predictors of outcome in all grades SAH patients, initial Hunt-Hess grade $(p=.018)$, periprocedural complications $(p=.014)$, external ventriculostomy $(E V D)$ presence $(p=.030)$ were significant predictors of unfavorable outcome, whereas age $(p=.009)$, rebleeding before treatment $(p=.002)$, EVD presence $(p=.003)$, delayed cerebral ischemia (DCI) $(\mathrm{p}=.035)$ were significantly associated with unfavorable outcome in the patients with good grade $\mathrm{SAH}$ who were defined as initial Hunt-Hess grade 1-3. On univariate and multivariate analysis for predictors of recurrence, aneurysm volume $(\mathrm{p}=.043)$, initial Raymond classification $(\mathrm{p}=.038)$ had a significant correlation with recurrence.

Conclusion The rate of unfavorable outcome in this study of SAH patients treated with endovascular method was $29.41 \%$ and the recurrence rate was $20.59 \%$. The present study demonstrates various predictors for unfavorable outcome and recurrence in the SAH patients treated with endovascular method. Therefore, careful individualized consideration in treatment for acute SAH patients who are planned to be treated with endovascular treatment is necessary.
Disclosures W. Yoon: None. W. Yoon: None. H. Roh: None. S. Suh: None. T. Kwon: None.

\section{E-039 ENDOVASCULAR FLOW-DIVERTING STENTS FOR INTRACRANIAL ANEURYSMS}

${ }^{1} \mathrm{~A}$ Nada*, ${ }^{1} \mathrm{M}$ Brunet, ${ }^{1} \mathrm{~S}$ Chen, ${ }^{1} \mathrm{~S}$ Sur, ${ }^{1} \mathrm{O}$ Elwardany, ${ }^{1} \mathrm{G}$ Zenonos, ${ }^{1} \mathrm{~A}$ Jahmshidi, ${ }^{1} \mathrm{D}$ MccCarthy, ${ }^{1} \mathrm{D}$ Sheinberg, ${ }^{2} \mathrm{~A}$ Zaher, ${ }^{2} \mathrm{M}$ Elsherbiny, ${ }^{2} \mathrm{M}$ Kassem, ${ }^{2} \mathrm{~S}$ Serag Eldin, ${ }^{2} \mathrm{~N}$ Mansour Ali, ${ }^{2} \mathrm{~A}$ Abd Elmoneim Ezz Eldin, ${ }^{1} \mathrm{~J}$ Thomson, ${ }^{1} \mathrm{D}$ Yavagal, ${ }^{1} \mathrm{E}$ Peterson, ${ }^{1} \mathrm{R}$ Starke. ${ }^{1}$ Neurological surgery, miami university, miami, FL; ${ }^{2}$ Neurological surgery, mansoura university, Mansoura, Egypt

\subsection{6/neurintsurg-2019-SNIS.114}

Background and purpose Flow-diverting devices now offer a treatment alternative for cerebral aneurysms. The concept of these devices is to promote thrombosis of aneurysms without filling the aneurysm cavity by deviating the blood flow away from the aneurysm neck which means treatment of the diseased segment harboring the aneurysm instead of treating the aneurysm itself. We present the results of a large single-center series of patients treated with the PED, including long-term follow-up.

Materials and methods Between January 2015 and February 2019, sidewall aneurysms with a neck ( $\geq 2 \mathrm{~mm}$ to $19 \mathrm{~mm}$ ) or unfavorable dome-neck ratio $(\leq 1.5)$; large/giant, fusiform, M1 dysplastic aneurysms, dissecting, blister like, and recurrent sidewall aneurysms; aneurysms at difficult angles; and aneurysms in which a branch was originating directly from the sac were treated with the PED. Patients were pre-medicated with dual antiplatelet medications. Data, including demographics, aneurysm features, clinical presentation, complications, results, and follow-up information are presented.

Results One hundred and fourteen aneurysms in 114 patients were treated. Of these, (100\%) PEDs were used for treatment. Adjunctive coiling was performed in 73 aneurysms (64\%). Symptomatic in-construct stenosis was detected in 1 patient $(0.9 \%)$ treated with percutaneous trans-arterial angioplasty. Any event rate as (Contrast extravasation and ICH, M1 occlusion or perforation, ICA occlusion, brain stem stroke, new $\mathrm{SAH}$ at the same side of treatment, ASDH, 3rd nerve palsy, Femoral pseudo aneurysm, breaking of delivery wire, snare of microwire, worsening of the double vision) was $9 / 114(7.8 \%)$, with a permanent morbidity of $4.3 \%$ and mortality of $0.9 \%$. Control angiography was available in 108 (94.7\%) patients. The aneurysm occlusion rate was $91.2 \%$ in 6 months, increasing to $94.6 \%$ in one year.

Conclusions Flow diverters allow for the endoluminal reconstruction of a parent artery for the treatment of complex and difficult-to-treat aneurysms. There are multiple devices on the market, but the PED is the most-studied and the only device with FDA approval. Published aneurysm occlusion rates are favorable with an acceptable adverse event rate when used for approved indications.

Disclosures A. Nada: None. M. Brunet: None. S. Chen: None. S. Sur: None. O. Elwardany: None. G. Zenonos: None. A. Jahmshidi: None. D. MccCarthy: None. D. Sheinberg: None. A. Zaher: None. M. Elsherbiny: None. M. Kassem: None. S. Serag Eldin: None. N. Mansour Ali: None. A. Abd Elmoneim Ezz Eldin: None. J. Thomson: None. D. Yavagal: None. E. Peterson: None. R. Starke: None. 